\title{
Straightening ventral curvature while preserving the urethral plate in proximal hypospadias repair
}

Snodgrass W, Prieto J

Department of Urology, Children's Medical Center, University of Texas Southwestern Medical Center at Dallas, Dallas, Texas, USA

J Urol. 2009; 182 (4 Suppl): 1720-5

Purpose: We report the efficacy of an expanded algorithm for penile straightening in proximal hypospadias surgery to preserve the urethral plate for urethroplasty. We also compared ventral corporotomy with grafting to multiple superficial ventral corporotomies without grafting for straightening greater than 30-degree ventral curvature.

Materials and Methods: The need for urethral plate transection was compared in 2 groups comprising consecutive patients with proximal shaft to perineal hypospadias repair done by one of us (WS). The 47 patients in group 1 underwent surgery from 2000 to 2005 and had ventral curvature greater than 30 degrees after degloving, leading to urethral plate transection, while in 23 in group 2 from 2006 to 2008 mobilization of the corpus spongiosum/urethral plate and proximal urethra were also performed before urethral plate transection. Patients in group 1 with greater than 30-degree ventral curvature after urethral plate transection underwent ventral corporotomy with grafting (7) or multiple transverse corporotomies without grafting (4), while those in group 2 
with greater than 30-degree ventral curvature after corpus spongiosum/urethral plate and urethral mobilization underwent multiple transverse corporotomies without grafting.

Results: Excluding 10 group 1 and 3 group 2 boys without ventral curvature after degloving the rate of urethral plate transection significantly decreased from $54 \%$ to $15 \%$ using the expanded algorithm $(\mathrm{p}=0.005)$. At a mean followup of 11 months in those with corpus spongiosum/urethral plate and urethral mobilization there was no recognized recurrent ventral curvature. Seven patients with greater than 30-degree ventral curvature underwent ventral corporotomy with grafting, while 11 underwent multiple transverse corporotomies without grafting. At a mean followup of 27 and 19 months, respectively, no patient had recurrent ventral curvature.

Conclusions: Mobilization of the corpus spongiosum/urethral plate and the urethra in proximal hypospadias cases with greater than 30-degree ventral curvature after penile degloving decreases the need for urethral plate transection. Ventral lengthening to correct corporeal disproportion can be achieved by corporotomy with grafting or by multiple transverse incisions without grafting.

\section{Editorial Comment}

Seventy patients with proximal shaft or scrotal hypospadias had preoperative testosterone therapy. From 2000-2005 those with less than $30^{\circ}$ of curvature, estimated by an artificial erection, were corrected by a dorsal plication. During that same time period, if the curvature was greater than $30^{\circ}$ the urethral plate was transected. From 2006-2008, if the curvature was greater than $30^{\circ}$, the urethral plate was mobilized. If after mobilization there was less than $30^{\circ}$ of curvature, then a midline dorsal plication was performed, while greater than $30^{\circ}$ curvature led to three transverse corporotomies in the region of greatest curvature on the ventrum without grafting and usually in combination with a single dorsal plication. The corporotomies were not deep enough to expose the corpora cavernosa tissue. A third technique included a ventral corporotomy with grafting that was done from the dermis. Of their 70 consecutive patients, after degloving the penis $19 \%$ had no curvature. Curvature less than $20^{\circ}$ was correctable by dorsal plication in $31 \%$ of the patients and in the $50 \%$ of patients with greater than $30^{\circ}$ one group had the urethral plate divided and the other group had plication plus the corporotomy incision or grafting. The outcome showed no difference in the followup. Only two cases in the early group had recurrent ventral curvature and none in the later group with preservation of the urethral plate.

It is interesting to note that corporotomies only without grafting in combination with plication and urethral mobilization was sufficient to correct curvature. It could be that grafting is unnecessary and it will be interesting to see if these patients over long-term growth and development continue to do so well. Hypospadias repairs based on the urethral plate enjoy a very good success rate and this shows that extended efforts to preserve the urethral plate may very well be worth it. In this era where two stage hypospadias repairs seem to be gaining in popularity, here is a technique to complete the hypospadias repair in one stage dealing with severe curvature at the same time. In simplistic terms, even if two stage repairs were entirely successful after two stages, they will never enjoy the same success rate as single stage repairs with followup surgeries to correct the complications.

Dr. Brent W. Snow

Division of Urology University of Utah Health Sci Ctr Salt Lake City, Utah, USA E-mail: brent.snow@hsc.utah.edu 